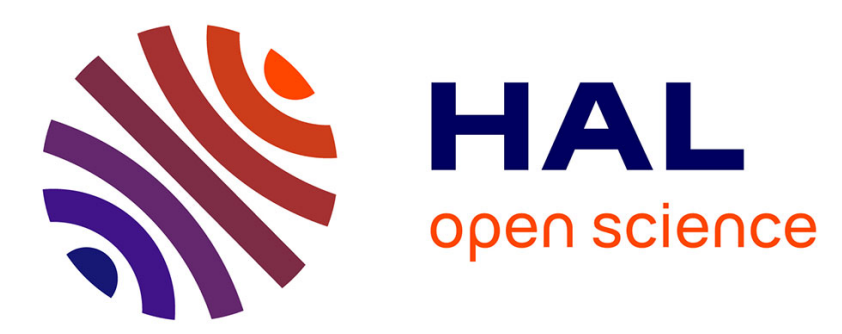

\title{
Système capacitif de mesure de la densité locale d'un fluide
}

\author{
J.P. Romagnan, J.P. Laheurte, J.C. Noiray, A. Gilabert
}

\section{To cite this version:}

J.P. Romagnan, J.P. Laheurte, J.C. Noiray, A. Gilabert. Système capacitif de mesure de la densité locale d'un fluide. Revue de Physique Appliquée, 1977, 12 (2), pp.465-468. 10.1051/rphysap:01977001202046500 . jpa-00244197

\section{HAL Id: jpa-00244197 https://hal.science/jpa-00244197}

Submitted on 1 Jan 1977

HAL is a multi-disciplinary open access archive for the deposit and dissemination of scientific research documents, whether they are published or not. The documents may come from teaching and research institutions in France or abroad, or from public or private research centers.
L'archive ouverte pluridisciplinaire HAL, est destinée au dépôt et à la diffusion de documents scientifiques de niveau recherche, publiés ou non, émanant des établissements d'enseignement et de recherche français ou étrangers, des laboratoires publics ou privés. 


\title{
SYSTĖME CAPACITIF DE MESURE DE LA DENSITÉ LOCALE D'UN FLUIDE
}

\author{
J. P. ROMAgnAN, J. P. LAhEURTe, J. C. NOIRAY et A. GILABERT \\ Laboratoire de Physique de la Matière Condensée \\ Université de Nice, Parc Valrose, 06034 Nice Cedex, France
}

(Reçu le 30 septembre 1976, accepté le 15 novembre 1976)

\begin{abstract}
Résumé. - Nous présentons un système capacitif permettant de détecter, dans un fluide macroscopique, des variations de densité locale se produisant à l'échelle de quelques couches atomiques. Ce système est étalonné à partir d'isothermes d'adsorption d' ${ }^{4} \mathrm{He}$.
\end{abstract}

\begin{abstract}
We describe a capacitive system able to detect local density variations occurring over a few atomic layers in a macroscopic fluid. This system has been calibrated from experimental ${ }^{4} \mathrm{He}$ adsorption isotherms.
\end{abstract}

1. Introduction. - Lorsqu'on s'intéresse aux propriétés locales d'un fluide, typiquement sur des épaisseurs de l'ordre de quelques couches atomiques, des techniques de mesure spécifiques sont nécessaires. Ces dernières années, de nouvelles techniques expérimentales se sont développées dans l'étude de films minces d'hélium liquide adsorbés sur une paroi. Citons les mesures de densité [1], de vitesse du son et d'ondes stationnaires acoustiques dans le film [2], et l'emploi de microbalance à quartz [3]. Ces techniques ont été déterminantes pour mieux comprendre la transition superfluide dans les films d ${ }^{34} \mathrm{He}[1,3]$, ou vérifier la théorie du champ de van der Waals créé par le substrat [2]. Malheureusement le domaine d'application de ces techniques est en général limité au cas des films minces.

Dans le cas de fluides de dimensions macroscopiques (quelques $\mathrm{mm}$ ou plus) les mesures locales sont aussi nécessaires lorsqu'on s'intéresse à l'influence du champ de van der Waals sur les couches atomiques du fluide se trouvant au voisinage immédiat de la paroi. Dans le cas de mélanges ${ }^{3} \mathrm{He}-{ }^{4} \mathrm{He}$ liquides par exemple, les gradients de concentration et de pression induits par le champ de van der Waals au voisinage des parois jouent un rôle fondamental sur la nucléation de la séparation de phase et de la transition $\lambda$ [4]. Le but de notre travail a été de mettre au point un système capacitif permettant de mesurer la densité locale d'un fluide aussi bien pour des films minces que pour des couches de fortes épaisseurs.

Dans la seconde partie nous décrivons le système mis au point et ses caractéristiques. Dans la troisième partie nous présentons les résultats obtenus pour des isothermes d'adsorption $\mathrm{d}^{\mathbf{4}} \mathrm{He}$. Enfin dans la quatrième partie nous présentons l'étalonnage de notre appareil.
2. Description du système. - Une variation de densité dans une couche de liquide d'épaisseur $e$ se traduit par une modification de la valeur de la constante diélectrique dans cette couche. Notre but est de détecter de façon capacitive des variations locales de densité dans des couches dont l'épaisseur $e$ est typiquement de l'ordre de quelques distances atomiques. Pour cela il faut satisfaire deux conditions essentielles.

D'une part pour que les variations relatives de capacité ne soient pas trop faibles, il faut que la contribution capacitive des lignes de champ dans la couche d'épaisseur $e$ ne soit pas négligeable devant la capacité totale du système ; c'est-à-dire qu'au moins une dimension du condensateur soit petite (quelques microns ou moins).

D'autre part la valeur absolue de la variation de capacité doit être expérimentalement détectable. Pour cela il faut que la valeur de la capacité totale, et par conséquent la surface des armatures soient suffisamment grandes (quelques centimètres carrés).

Ces deux conditions sont très difficiles à satisfaire en utilisant la technique d'un condensateur plan classique constitué de deux plaques métalliques séparées par des espaceurs en mica [5]. Par contre les techniques d'évaporation sous vide et d'attaque photochimique sont particulièrement bien adaptées à l'élaboration de structures dont les dimensions sont à l'échelle du micron. En utilisant ces techniques nous avons réalisé un ensemble de 5000 condensateurs en parallèle formant une structure en peigne dont la longueur développée est de $100 \mathrm{~m}$ (Fig. 1). La capacité de l'ensemble sous vide est de $2960 \mathrm{pF}$ à $2,36 \mathrm{~K}$. Nous avons procédé à partir de plaques de verre [6] recouvertes d'une couche de chrome évaporé sous vide d'épaisseur $h=800 \AA$. L'attaque photochimique, extrêmement délicate étant donné la surface importante de notre échantillon 


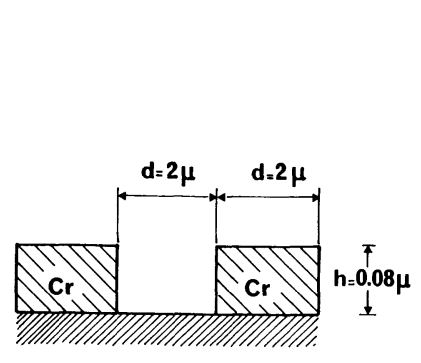

Substrat en verre

(a)

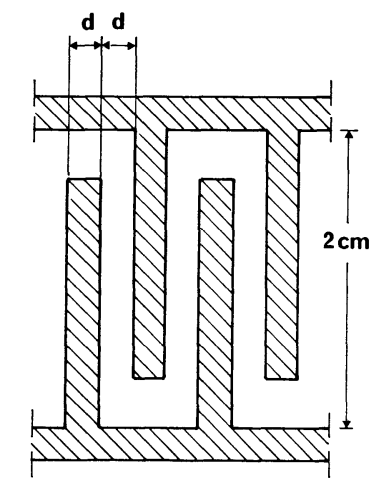

(b)

FIG. 1. - Géométrie du système capacitif $a$ ) Vue en coupe ; b) Vue de dessus. Les dimensions ne sont pas à l'échelle.

$\left(4 \mathrm{~cm}^{2}\right)$, et le masque photographique qui nécessite de nombreuses photorépétitions avec une tolérance de raccordement inférieure à un micron, ont été exécutés par le Centre Electronique Horloger de Neufchâtel [7].

Nous n'avons pas pu diminuer la valeur de la distance $d$ au-dessous de 2 microns à cause de nombreuses difficultés technologiques. La principale est liée au fait que les armatures du condensateur doivent être isolées électriquement entre elles. C'est-à-dire qu'à la fin de l'attaque photochimique il ne doit pas subsister de petits ponts métalliques reliant entre elles deux électrodes consécutives. Ces ponts sont dus à la présence de grains de poussière lors de l'enduction ou de l'insolation de la résine photosensible. Sur une surface aussi importante que la nôtre $\left(4 \mathrm{~cm}^{2}\right)$ il est très difficile, malgré de multiples précautions, de s'affranchir complètement de la présence de ces grains de poussière, dont le nombre est inversement proportionnel à la taille [8]. Dans notre cas il est clair que seuls les grains dont la taille est supérieure à deux microns seront gênants. A la fin de l'attaque photochimique, un examen complet sous microscope de notre échantillon nous a permis de localiser quatre courts-circuits. Nous les avons éliminés par une nouvelle attaque photochimique, l'insolation étant réalisée sour microscope à l'aide d'un faisceau collimaté.

Par ailleurs, nous détectons les variations de capacité à l'aide d'un pont dont le signal d'attaque est de $10 \mathrm{~V}$, ce qui, pour $d=2 \mu$, donne un champ électrique de $5 \times 10^{4} \mathrm{~V} / \mathrm{m}$. Cette valeur est voisine de celle du champ disruptif de l'hélium, par conséquent diminuer $d$ conduirait à abaisser le niveau du signal d'attaque et n'améliorerait pas la sensibilité de détection du système.

Le pont que nous utilisons est un pont Général Radio 1615 A dont la tension de déséquilibre est détectée par un PAR 124 utilisé en voltmètre. L'affichage de la valeur de la capacité fournie par le pont comporte six chiffres. En lui ajoutant une capacité extérieure de $10^{-2} \mathrm{pF}$, mise en circuit par un diviseur variant de 0 à 1 par bonds de un dixième, on obtient un septième chiffre. Nous pouvons ainsi détecter des variations relatives de capacité de $10^{-6}$.

Enfin nous avons vérifié que les résultats de nos mesures étaient indépendants du niveau du signal d'attaque entre 1 et $10 \mathrm{~V}$.

3. Isothermes d'adsorption $d^{\mathbf{4}} \mathbf{H e}$ - Nous présentons ici les résultats de trois expériences d'adsorption $\mathrm{d}^{34} \mathrm{He}$ réalisées à trois températures : $2,36 \mathrm{~K}$, $2,13 \mathrm{~K}$ et $1,95 \mathrm{~K}$.

Nous mesurons d'abord la valeur $C_{0}$ de la capacité sous vide à la température $T$ de l'expérience. Puis nous effectuons une entrée d'hélium gazeux sous une pression $p$ inférieure à sa pression de vapeur saturante $p_{0}(T)$ à cette température. Du fait du potentiel d'interaction de van der Waals un certain nombre d'atomes d'hélium sont adsorbés sur la paroi, formant une couche solide recouverte d'une couche liquide (Fig. 2a). La distance $e$

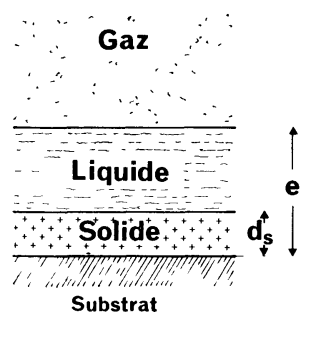

(a)

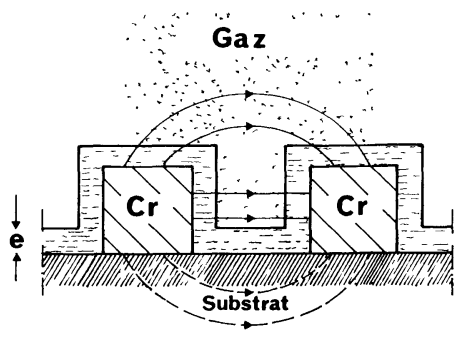

(b)
FIG. 2. - a) Représentation schématique de l'adsorption d'atomes d'hélium sur une paroi.

b) Nous avons représenté en traits pleins les lignes de champ dont la contribution capacitive est affectée par le fluide adsorbé sur la paroi et par le gaz. Les lignes de champ représentées en pointillé se propagent dans le substrat en verre. leur contribution capacitive est indépendante du fluide.

entre la paroi et la surface libre du liquide est donnée par [9] :

$$
e=\left[\frac{T}{\theta} \log \frac{p_{0}}{p}\right]^{-1 / 3}
$$

$e$ est exprimé en couches atomiques ( 1 couche atomique $=3,6 \AA$ ) $\theta$ paramètre d'interaction de van der Waals est pris égal à $27 \mathrm{~K}$ [10].

Nous avons représenté figure 3 la variation de $e$, calculée d'après (1), lorsque $p$ augmente de 0 à $p_{0}$.

Soit $C(p)$ la valeur mesurée de la capacité pour une pression $p$. La variation de capacité $\Delta C(p)=C(p)-C_{0}$ résulte de la présence d'une part d'atomes d'hélium adsorbés sur la paroi, et d'autre part d'atomes d'hélium à l'état de gaz (Fig. $2 b$ ).

Nous avons représenté figure 4 les variations de $\Delta C(p)$ en fonction de la pression pour trois températures différentes. Les trois isothermes présentent le même comportement caractéristique.

D'abord une brusque variation de $\Delta C(p)$ à très faible pression traduisant la formation d'une première couche adsorbée. Nous constatons que les valeurs de $\Delta C$ obtenues en extrapolant linéairement nos courbes 


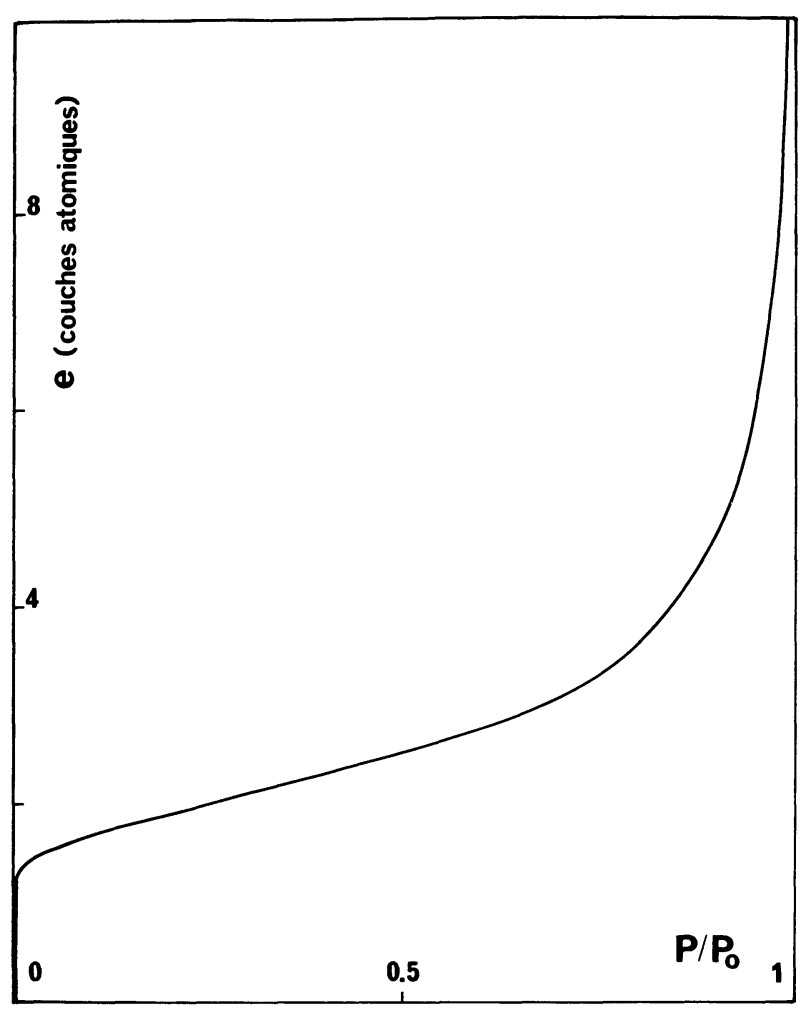

Fig. 3. - Variation de la distance $e$ entre la paroi et la surface libre du liquide calculée d'après l'équation (1) pour $T=2,36 \mathrm{~K}$ et $\theta=27 \mathrm{~K} ; p_{0}$ est la pression de vapeur saturante à la température considérée.

jusqu'à $P=0$ sont identiques dans la limite de la précision de nos mesures.

Nous observons ensuite une région de variation linéaire jusqu'à environ $0,7 p_{0}$. Dans cette zone, l'épaisseur du film d'hélium liquide varie peu avec la pression (Fig. 3), et c'est l'hélium gazeux qui fournit la contribution essentielle à la variation de capacité $\Delta C(p)$. Or la constante diélectrique de l'hélium gazeux s'écrit [11] :

$$
\varepsilon_{\mathrm{g}}-1=\frac{1,547}{V_{\mathrm{m}}}
$$

$V_{\mathrm{m}}$ volume molaire en $\mathrm{cm}^{3}$ de l'hélium gazeux à la température et à la pression de l'expérience.

C'est-à-dire que $\varepsilon_{\mathrm{g}}-1$ varie linéairement avec la pression.

Enfin lorsque la pression devient voisine de $p_{0}$ l'épaisseur du film liquide croît très fortement (Fig. 3) et $\Delta C(p)$ augmente très rapidement jusqu'à la valeur $\Delta C\left(p_{0}\right)$ qui correspond à la saturation de la capacité en hélium liquide. A partir de cette valeur $\Delta C\left(p_{0}\right)$ nous pouvons calculer la contribution capacité $C_{\mathrm{s}}$ des lignes de champ supérieure (Fig. 2b). En effet :

$$
\Delta C\left(p_{0}\right)=C_{\mathrm{s}}\left(\varepsilon_{1}-1\right)
$$

$\varepsilon_{1}$ constante diélectrique de l'hélium liquide.

Nous trouvons à $2,36 \mathrm{~K} C_{\mathrm{s}}=451,8 \mathrm{pF}$ soit $15 \%$ de la capacité totale $C_{0}$. La contribution capacitive la plus importante est donc celle due aux lignes de champ

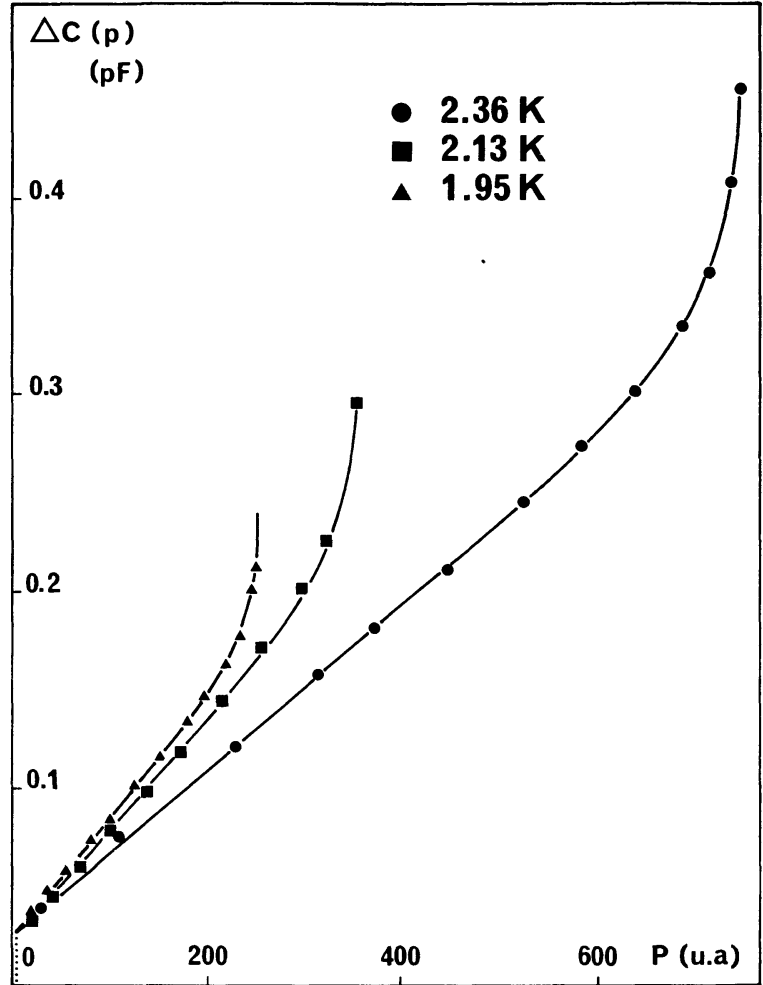

FIG. 4. - Isothermes d'adsorption d'4He. Nous avons représenté en tirets la partie de la courbe correspondant aux très faibles pressions.

se propageant dans le verre. Cette contribution constitue à température constante un fond continu indépendant du fluide étudié.

Les valeurs de $\Delta C\left(p_{0}\right)$ trouvées dans nos trois expériences reflètent bien les variations de la densité de $1{ }^{4} \mathrm{He}$ dans ce domaine de température.

4. Etalonnage du système. - Lorsqu'une variation de densité se produit dans une couche liquide d'épaisseur moyenne $e$ la modification correspondante $\Delta \varepsilon$ de la constante diélectrique entraîne une variation de capacité

$$
\Delta C=\Delta \varepsilon f(e)
$$

où $f(e)$ est un facteur géométrique représentant la contribution capacitive des lignes de champ dans la couche d'épaisseur $e$.

L'étalonnage de notre système consiste à déterminer les valeurs de $f(e)$ en fonction de $e$ à partir des isothermes d'adsorption précédentes. A une pression $p$ donnée, la variation de capacité mesurée $\Delta C(p)$ résulte de la présence sur le substrat et sur les électrodes d'une couche solide d'épaisseur moyenne $d_{\mathrm{s}}$, d'une couche liquide d'épaisseur moyenne $e-d_{\mathrm{s}}$, et de gaz (Fig. 2). Les dimensions $e$ et $d_{\mathrm{s}}$ étant très petites (quelques dizaines d'angstroems) devant $d(2 \mu)$, on peut au premier ordre linéariser $f(e)$ et $\Delta C(p)$ :

$f(e)=a e$

$\Delta C(p)=\left(\varepsilon_{\mathrm{g}}-1\right) C_{\mathrm{s}}+\left(\varepsilon_{1}-\varepsilon_{\mathrm{g}}\right) a e+\left(\varepsilon_{\mathrm{s}}-\varepsilon_{1}\right) a d_{\mathrm{s}}$ 
$e$ et $\varepsilon_{g}$, définis respectivement par les équations (1) et (2), varient avec la pression $p$.

En négligeant la faible dépendance en pression de $\varepsilon_{\mathrm{s}}$ et $\varepsilon_{1}$ et en les considérant comme constants dans le domaine restreint de température de nos expériences nous avons : $\varepsilon_{1}=1,056$ et $\varepsilon_{\mathrm{s}}=1,077$.

La valeur maximale de $\varepsilon_{\mathrm{g}}$ dans nos expériences étant 1,0006 on peut écrire : $\varepsilon_{1}-\varepsilon_{\mathrm{g}} \simeq \varepsilon_{1}-1=0,056$.

L'équation (6) devient alors :

$$
\Delta C(p)-\left(\varepsilon_{\mathrm{g}}-1\right) C_{\mathrm{s}}=0,056 a e+0,021 a d_{\mathrm{s}} .
$$

Le membre de droite de l'expression (7) est indépendant de la température. A partir des valeurs expérimen-

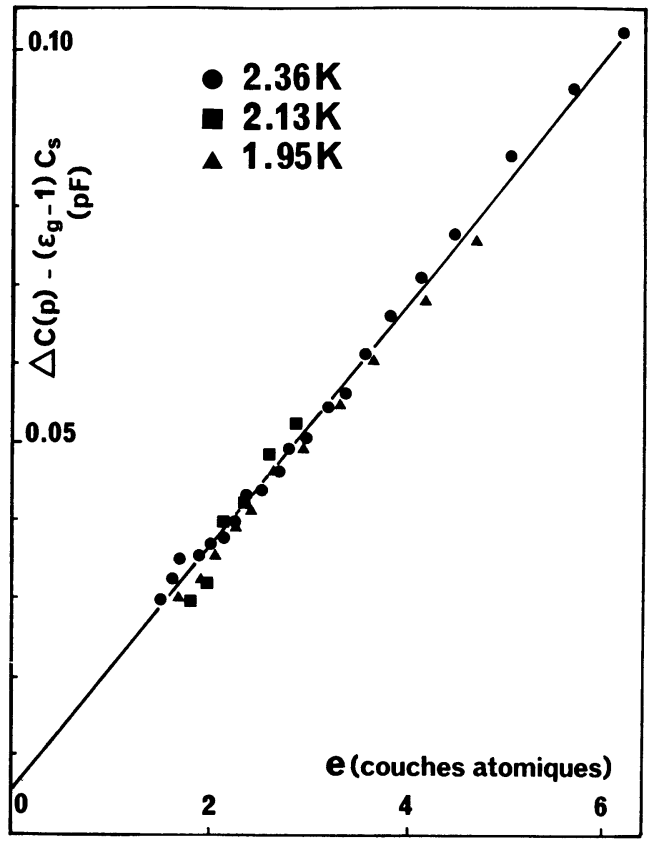

Fig. 5. - Variations de $\Delta C(p)-\left(\varepsilon_{\mathrm{g}}-1\right) \mathrm{C}_{\mathrm{s}}$ en fonction de $e$ obtenues à partir des isothermes d'adsorption. Le comportement linéaire obtenu confirme la validité de l'équation (7). tales de $\Delta C(p)$ (Fig. 4), et des valeurs de e et $\varepsilon_{\mathrm{g}}$ calculées d'après (1) et (2), nous avons représenté les variations de $\Delta C(p)-\left(\varepsilon_{\mathrm{g}}-1\right) C_{\mathrm{s}}$ en fonction de $e$ (Fig. 5). Les points obtenus sont bien alignés sur une droite ce qui confirme la validité des expressions linéarisées utilisées en (5) et (6) [13]. La pente de cette droite et son intersection avec l'axe des ordonnées nous permettent de calculer $d_{\mathrm{s}}$ et $a$. Nous avons trouvé $d_{\mathrm{s}}=1,03$ couche atomique, ce qui est en bon accord avec le résultat d'autres expériences [1], et $a=0,28$. Par conséquent une modification $\Delta \varepsilon$ de la constante diélectrique dans une couche d'épaisseur $e$ au voisinage des parois entra ${ }_{\mathrm{s}}$ ne une variation de capacité de notre système :

$$
\Delta C=0,28 \text { e } \Delta \varepsilon
$$

$\Delta C$ en picofarads,

$e$ en couches atomiques.

La limite de résolution de notre technique de mesure est de $2 \times 10^{-3} \mathrm{pF}$, ce qui correspond par exemple à la détection d'une variation de constante diélectrique $\Delta \varepsilon=10^{-2}$ dans une couche atomique. Il est clair que l'utilisation d'une technique de mesure de capacité possédant une meilleure résolution [12] améliorerait encore la sensibilité de détection du système.

5. Conclusion. - Nous avons présenté un système capacitif permettant de détecter des variations locales de densité aussi bien pour des films minces que pour des couches de fortes épaisseurs ; nous l'avons étalonné à partir d'isothermes d'adsorption d'un fluide de densité connue $\left({ }^{4} \mathrm{He}\right)$. D'une façon générale ce système trouve son application dans l'étude des phénomènes d'adsorption et des effets de surface. Notamment un prolongement direct de ce travail est l'étude de l'influence des parois sur la nucléation de la séparation de phase dans des mélanges liquides ${ }^{3} \mathrm{He}-{ }^{4} \mathrm{He}$ à basse température.

\section{Bibliographie}

[1] Wang, T. G. and Petrac, D., Phys. Rev. B 11 (1975) 4221.

Williams, G. A. and Packard, R. E., Phys. Rev. Lett. 32 (1974) 587.

[2] Scholtz, J. H., Mc Lean, E. D. and Rudnick, I., Phys. Rev. Lett. 32 (1974) 150.

SABISKy, E. S. and ANDERson, C. H., Phys. Rev. A 7 (1973) 750.

Frenois, Ch., Joffrin, J., Legros, P. and Levelut, A., Phys. Rev. Lett. 32 (1974) 1295.

[3] Chester, M. and Yang, L. C., Phys. Rev. Lett. 31 (1973) 1377.

[4] Chester, M., Laheurte, J. P. and Romagnan, J. P. (à paraître dans Phys. Rev.).
[5] Laheurte, J. P., Phys. Rev. 6A (1972) 2452.

[6] Plaques au chrome Balzers type L. R. C. 300.

[7] Centre Electronique Horloger 2001 Neuchatel (Suisse).

[8] Kodak Photoresist Seminar Proceedings, Volume II (1968).

[9] Rudnick, I., Kagiwida, R. S. and Fraser, J. C., Phys. Rev. Lett. 20 (1968) 430.

[10] Sabisky, E. S. and Anderson, C. H., Phys. Rev. Lett. 30 (1973) 1122.

[11] Watson, G. E., Reppy, J. D. and Richardson, R. C., Phys. Rev. 188 (1969) 384.

[12] Dandache, H., Briggs, A. et Papoulard, M. (à paraître).

[13] Pour les films épais, correspondant à de grandes valeurs de $e$, il faut disposer d'un appareil de mesure de pression de résolution meilleure que $10^{-3}$. 